\title{
Motivational influences on cognitive control: Behavior, brain activation, and individual differences
}

\author{
HANNAH S. Locke ANd TODd S. BRaVer \\ Washington University, St. Louis, Missouri
}

\begin{abstract}
What changes in brain activity are associated with changes in motivational state? The present study addressed this question by having participants perform a cognitive task (AX variant of the Continuous Performance Test; AX-CPT) under three different blocked motivational conditions (reward-incentive, penalty-incentive, and baseline). Behavioral data showed that the incentive conditions modulated task performance, potentially by altering participants' cognitive control strategy. Neuroimaging data indicated that the reward condition was associated with a sustained increase in a primarily right-lateralized network that included parietal and prefrontal cortex. Additionally, individual differences were observed, such that activation in both reward-related brain regions and frontopolar cortex was linked to the degree of motivation-induced performance enhancement and to motivation-related personality variables. These results suggest that changes in motivational state may modulate performance through sustained activity in cognitive control regions and that the effect of incentives may be affected by the personalities of the participants.
\end{abstract}

It is a truism of cognitive research that participants perform experimental tasks with varying levels of motivation. Some participants appear to show little interest and exert minimal effort in their task performance, whereas others seem to approach the task as a critical test, exhibiting a burning desire to perform to their utmost ability. Yet, even though this variation in motivation is a well-known phenomenon, it seems to be underappreciated and underexplored. One source of variation might be a participant's motivational state while performing the task. A natural way to explore such a hypothesis is to experimentally manipulate motivational states - for example, by providing performance-based incentives - to assess their impact on cognitive performance. That is, by placing motivational states under experimental control, one can examine how such state-related effects affect how cognitive tasks are performed.

It is also important to appreciate that providing a reward does not automatically and consistently instill motivation in participants, since individuals may differ in their reactions to rewards. Thus, a second source of variation might be individual differences in motivation-related traits, such as sensitivity to different types of incentives. By measuring such types of trait variation, one can also estimate the impact on cognitive performance of stable individual differences in motivationally relevant variables. Currently, such motivationally oriented individual difference studies are almost nonexistent in the cognitive literature.

Yet, even if motivational states are so experimentally controlled that all participants perform a task with equally strong motivation, whether or not increased motivation per se is sufficient to enact a change in task-relevant components of cognitive processing, so as to improve performance, is a separate question. Mere wanting, without appropriate directed action, does not imply obtainment. Thus, a third source of variation might be the efficacy of the pathway that translates increased motivation into optimized cognitive processing.

Neuroimaging techniques provide a particularly promising approach to understanding motivation by showing how variation in motivation-related states and traits affect the neural circuitry engaged during cognitive task performance. With this insight, it is possible to gain a greater understanding into the causal pathway where motivation affects cognition and thereby translates into changes in observable behavior. The goal of the present study was to explore this question by using functional magnetic resonance imaging (fMRI) to examine the following points: (1) whether reward-focused motivational states produce sustained changes in brain activity and behavioral performance, and (2) whether such motivational manipulations show important influences related to individual differences.

There has been a recent upsurge in attention in the cognitive neuroscience literature on the impact of reward incentives on brain activity and behavior. A wealth of imaging studies have identified brain regions responsive to reward, in the signaling, prediction, and representation of incentives (for reviews, see Breiter \& Rosen, 1999; O'Doherty, 2004). However, what a motivational state entails, and how motivational systems in the brain interact with other brain areas to contribute to improving performance, re- 
mains a gap in the literature that has only recently begun to be addressed (e.g., Gilbert \& Fiez, 2004; Pochon et al., 2002; Small et al., 2005; Taylor et al., 2004).

Recently, a few studies have looked specifically at reward and cognition interactions in the brain to discover the neural mechanisms by which an increase in motivation leads to performance gains. Pochon et al. (2002) demonstrated an increase in activity in regions associated with executive control in humans during a reward condition. Comparing activity in an $n$-back task when a reward was offered with activity when no reward was offered yielded an increase in activity in dorsolateral prefrontal cortex (DLPFC), an area involved maintaining task set (Miller \& Cohen, 2001). In their study, both increases in task demand and reward increased activity in DLPFC. In this way, reward may act as a kind of call for increased effort in a similar way as a change in actual task demands. In both instances, more effort is required to maintain or improve behavior.

Interestingly, Pochon et al. (2002) also reported the recruitment in response to reward of lateral frontopolar regions, which are associated with the need to maintain an overall goal while simultaneously performing a task with its own local set of subgoals (Koechlin, Basso, Pietrini, Panzer, \& Grafman, 1999). According to Pochon et al., reward - or what is at stake at any given moment - may be represented in the form of a primary, high-order goal in the frontopolar region. This goal may then coordinate the processing and management of subgoals that enable optimal completion of the task.

Results from Taylor et al. (2004) showed an increase in activation in task-relevant regions in an object working memory study. In their study, they were able to decompose the trials into discrete phases, and during the delay phase they found increased activity in the premotor superior frontal sulcus and the intraparietal sulcus. These areas, involved in maintenance of information, also increased in response to working memory load. This suggests that, as in Pochon et al. (2002), reward may elicit enhanced processing in the same way as an increase in task demands. Taylor et al. also found activity in a reward processing region of the basal ganglia, the caudate nucleus, which was active during trials involving reward and was not modulated by the discrete trial events.

The findings outlined above provide some intriguing answers to how reward information is used in the brain to improve performance. Results so far show that the brain systems associated with reward, with cognitive control structures, and with the individual tasks themselves, all show increased activity under reward conditions, sometimes in the same way as for increased task demands. However, as knowledge grows about the regions involved in the motivation and cognition interactions, it is also important to consider the temporal dynamics of the relationship between motivation and cognition.

Specifically, short bursts of effort may not be effective, since many tasks require consistent effort and concentration to maximize reward. For example, maintaining vigilance during intertrial intervals rather than lapsing into reverie may make participants faster to perceive and respond to stimuli when they appear. Additionally, most of the previous imaging research focused on reward processing per se has not required behavioral improvements to increase rewards, and as such cannot address questions about the mechanisms by which a motivational state might improve behavioral performance. Furthermore, many previous studies looked at reward on a trial-by-trial basis, investigating the transient adjustments in activity by which the brain tracks rewards. A motivational state, by contrast, may be associated with a more stable, heightened pattern of activity in regions associated with cognitive control, or task performance, that endures for the duration of the task.

The present study examined brain activation during cognitive task performance, under different motivational states, induced by monetary incentives. The primary goal was to investigate how incentives affect performance, and to determine the neural correlates of these state-related performance changes. A particular emphasis was placed on looking for regions that were active in a sustained manner, as these might be relevant to motivational state as well as to the task demands that are needed consistently rather than transiently. To address this question, this study used a mixed state-item design for sustained or tonic activity to be dissociated from transient or trial-specific activation. In particular, this technique enables isolation of sustained changes in activity associated with experimental manipulations of motivation state, over and above any transient or event-related effects that might be produced by such manipulations (e.g., Braver, Reynolds, \& Donaldson, 2003).

Although transient effects of reward motivation are interesting per se, there have been previous studies examining such effects (e.g., Delgado, Locke, Stenger, \& Fiez, 2003; Delgado, Nystrom, Fissell, Noll, \& Fiez, 2000). However, to our knowledge, no previous study has examined whether sustained changes in neural activity are produced as a result of a change in reward-oriented motivational states. Moreover, examinations of transient effects of reward motivation on cognitive task performance can be better conducted in studies in which reward is manipulated in a trial-by-trial manner (e.g., parametrically), as has been investigated in previous studies (e.g., Taylor et al., 2004).

We predicted that the motivational manipulation would enhance sustained activity in brain regions engaged in cognitive control processes. To more specifically examine this question, we utilized an experimental task-a variant of the continuous performance test (CPT), the AX-CPT (Rosvold, Mirsky, Sarason, Bransome, \& Beck, 1956) - that has been used as a selective probe of cognitive control processes in many previous behavioral and neuroimaging studies (Braver, Barch, \& Cohen, 1999; Carter et al., 1998). At the behavioral level, particular profiles of performance can reveal different cognitive control strategies, which may provide a good diagnostic of the effects of motivational manipulations. In terms of neural substrates, previous studies have shown that cognitive control in the AX-CPT is associated with sustained lateral PFC activity (Barch et al., 1997; Braver \& Bongiolatti, 2002; Braver, Cohen, \& Barch, 2002), which may reflect the active maintenance of task-set information (Miller \& Cohen, 2001). This same region has also previously been implicated in motivational manipulations (Pochon et al., 2002; 
Watanabe, Hikosaka, Sakagami, \& Shirakawa, 2002), and so was a particular focus of interest here. Given previous findings suggesting right lateral PFC activity associated with sustained attentional control processes (Pardo, Fox, \& Raichle, 1991; Posner \& Petersen, 1990), we specifically predicted that the PFC activation associated with reward motivation would be right-lateralized.

Participants performed the AX-CPT task under blocked reward-incentive and no-incentive conditions, so the incentive was constant across trials. The criterion for reward obtainment was individually calibrated for each participant on the basis of his or her baseline performance, to achieve an expected reward rate of $50 \%$. This approach allowed for maximal sensitivity to motivation-induced performance changes under reward incentive conditions, while also providing a means of both controlling for, and assessing, individual differences in baseline performance and in motivation-induced changes in performance. We predicted that the reward incentive would be effective in improving behavioral performance, by generally speeding reaction time (RT) without increasing overall error rate.

Although the main focus of the current study was to look at how motivation for reward affects behavior and brain activity, a penalty condition was also included, in which participants could lose money for committing a specific type of error: failing to withhold responding on a no-go trial. The penalty condition was not intended to provide a tightly controlled contrast to the reward condition. Instead, this condition was treated as an additional control for more nonspecific effects of motivational changes due to financial consequences on performance, such as increased arousal or greater task engagement and attention. Although effects of negative incentives on performance are of interest in their own right, and will form the basis of a forthcoming report, here we focus primarily on the behavioral and neural changes associated with rewardfocused motivational states.

A final consideration, often overlooked in neuroimaging research, is that there may be substantial individual differences in how motivational incentives are processed, or in the ability of such incentives to modulate behavior. There is a large personality literature and a small but growing cognitive neuroscience literature that suggests substantial individual differences in the way that individuals respond to reward or cues for reward in the environment. Several well-established scales have been developed to measure such dimensions of individual difference, including the Behavioral Inhibition/Approach System (BIS/BAS; Carver \& White, 1994) and the Generalized Reward and Punishment Expectancy Scale (GRAPES; Ball \& Zuckerman, 1990). The scales are similar, but also tap into somewhat different processes; the BAS subscale of the BIS/BAS measures sensitivity to reward cues and their effects on emotional and behavioral responses, whereas the reward subscale of the GRAPES taps into longer term expectations regarding the likelihood of rewards being obtained. It is plausible that individual differences in reward sensitivity or expectancy, as measured by scales such as the BIS/BAS and GRAPES, could affect reward processing during motivational con- texts, which could affect cognitive control, task-relevant processing and, ultimately, behavior.

Previous imaging studies that incorporated scores on the BAS scale have shown that activity in regions like the anterior cingulate (Gray \& Braver, 2002) and the left lateral PFC (Gray et al., 2005) are correlated with BAS. Specifically, higher BAS scores were associated with lower activity, despite equivalent performance with the low BAS group, suggesting greater processing efficiency. However, thus far neither the BAS nor GRAPES has been studied in an imaging study concerned with motivation. Yet, given their relevance to reward, it seems plausible that individual differences in either or both dimensions may modulate brain activity in reward-related regions, such as those in the ventral and dorsal striatum and orbitofrontal cortex, or in cognitive control regions, such as the lateral $\mathrm{PFC}$ and anterior cingulate. Nevertheless, it is difficult to make specific predictions, due to the scarcity of previous work on individual differences in the brain. Our intent was to perform exploratory analyses in order to generate new specific hypotheses that could be more directly tested in follow-up studies.

\section{METHOD}

\section{Participants}

Twenty volunteer participants were recruited from Washington University to participate in return for payment $(\$ 25 / \mathrm{h})$. All participants were right-handed native English speakers, and were free from psychiatric or neurological disorders. Written informed consent was obtained, and the study was approved by the Washington University Medical Center human subjects committee. Each participant was also screened for any physical or medical condition affecting eligibility for fMRI prior to being scanned. Four participants were excluded from analysis because of incomplete data, excessive movement, or technical problems at the time of scanning. Thus, primary analyses focused on the remaining 16 participants ( 7 male), age range, 18-28. An additional participant did not complete the personality measures, so analyses of individual differences only included 15 participants.

\section{Procedure}

While undergoing fMRI scanning, participants engaged in a continuous performance task, the AX-CPT, which was specifically designed to measure cognitive control in terms of the ability to utilize and maintain contextual cue information to bias attention and action. Each AX-CPT trial lasted $7.5 \mathrm{sec}$, and consisted of a contextual cue $(300 \mathrm{msec})$, a delay period $(4,900 \mathrm{msec})$, a probe $(300 \mathrm{msec})$, a second delay $(1,000 \mathrm{msec})$, and a feedback screen indicating performance on the trial $(1,000 \mathrm{msec})$. Participants were instructed to press the target button only when a specific probe item, X, occurred immediately following a particular contextual cue, A (hence the "AX" in the task name). Target AX trials occur with high frequency (70\%), creating specific expectancies and response tendencies that can be examined, such as on AY trials $(10 \%)$ that feature the usually reliable cue A but not the X probe, and on BX trials (10\%) that have the $\mathrm{X}$ probe without the necessary A cue.

Relative performance on these two types of nontarget trials, compared with the additional BY nontarget control condition (10\%), provides an index of two types of cognitive control strategy: proactive and reactive control (for more information on the dual mechanisms of control theory, see Braver, Gray, \& Burgess, 2007; Braver, Satpute, Rush, Racine, \& Barch, 2005). Proactive control involves utilizing actively maintained context information to prime response selection. Thus, participants using a proactive control strategy would keep the cue letter actively in mind during the delay, while waiting for the probe letter to appear. Proactive control should result in rela- 
tively good performance on BX (and AX) trials, because in these two trial types, the cue letter accurately predicts which response to make (nontarget for B, $100 \%$ of the time; target for A, $87.5 \%$ of the time). However, proactive control yields poorer AY trial performance, since on these trials participants' prior experience leads them to predict a target response.

In contrast, reactive control involves utilizing context information only when needed. Thus, participants using reactive control reactivate the context at the time of the probe by retrieving it from memory. Reactive control should result in poorer performance on $\mathrm{BX}$ trials, because the $\mathrm{X}$ probe is associated most strongly with a target response, which results in a competing response association when the cue is actually retrieved, but better AY performance, because letters other than X prompt a (correct) nontarget response.

The AX-CPT task was performed in three conditions: baseline, reward, and penalty. Participants performed a total of 120 trials in each condition. The baseline condition had standard frequencies of each trial type $(\mathrm{AX}=70 \%, 84$ trials; $\mathrm{AY}, \mathrm{BX}, \mathrm{BY}=10 \%, 12$ trials each). In the reward and penalty blocks, $80 \%$ of the trials were as above, with approximately the same frequency rates as in the baseline condition (60 AX trials, plus 12 each of the $\mathrm{AY}, \mathrm{BX}$, and $\mathrm{BY}$ ). The remaining $20 \%$ of trials ( 24 total) were no-go trials, indicated by an underlined probe letter, which required participants to withhold their response. These no-go trials were included for the purposes of the penalty incentive condition, described below, and were not predicted to have a strong impact on AX-CPT performance, other than by slowing overall RT. Moreover, any RT slowing caused by the inclusion of no-go trials in the reward condition relative to baseline should have reduced any tendency to observe any reward-related improvements in task performance.

Participants performed the baseline condition first, without any instruction that future blocks would be performed with financial incentives. The next two conditions were the reward and penalty blocks, which were performed in counterbalanced order across participants. During reward blocks, 25 cents was offered for each trial faster than the participant's median RT in the baseline blocks. The default reward rate if performance remained unchanged would be $50 \%$, so there was room for significant improvement. During penalty blocks, participants were told that $\$ 3$ would be subtracted from their payment for each no-go trial on which they failed to withhold their response. Feedback regarding reward or punishment was given following each trial. A general feedback message was also given in the baseline blocks, to control for visual information. Contrary to what they were told, at the end of the scanning session each participant was awarded payment in full, plus $\$ 25$, a bonus of slightly over the maximum possible reward.

In a separate session, participants filled out a large packet of personality measures. Of particular interest to this study are the measures dealing with reward, specifically reward expectation (GRAPES; Ball \& Zuckerman, 1990) and reward sensitivity (BIS/ BAS; Carver \& White, 1994). The BAS construct is thought to reflect an individual's reactivity to cues of reward. It uses statements like "It would excite me to win a contest" to quantify how interested in potential rewards participants are: Highly reward-sensitive people tend to find rewards gratifying, and will expend significant effort to achieve them. The GRAPES (reward subscale) is thought to tap into a slightly different aspect of reward processing than does BAS. It uses statements like "If I invested in stocks, I would probably make money." A high score on the GRAPES indicates a kind of optimism, whereby people believe that they are likely to obtain rewards available in the environment. These two personality scales are conceptually related, because it would be difficult to be highly excited by reward cues if one felt there were little chance of achieving the reward. GRAPES may be more specific, however, in attuning to one particular element of how people perceive reward: achievability. The similarity, but also the separateness, of the constructs is confirmed by testing with a large sample of participants $(N=268)$, in which a moderate correlation of $r=.375$ was obtained (Locke \& Braver, unpublished results). In the present sample, the two measures were correlated at $r=.17$ (n.s.).

\section{fMRI Acquisition}

Images were acquired on a Siemens 1.5 Tesla Vision System (Erlangen, Germany) with a standard, circularly polarized head coil. A pillow and tape were used to minimize head movement. Headphones dampened scanner noise and enabled communication with participants. Both structural and functional images were acquired at each scan. High-resolution $(1.25 \times 1 \times 1)$ structural images were acquired using a sagittal MP-RAGE 3D T1-weighted sequence $(\mathrm{TR}=$ $9.7 \mathrm{~mm}, \mathrm{TE}=4$, flip $=12^{\circ}$, TI $=300 \mathrm{msec}$ ) (Mugler \& Brookeman, 1990). Functional images were acquired using an asymmetric spin-echo echo-planar sequence $(\mathrm{TR}=2,500, \mathrm{TE}=50 \mathrm{msec}$, flip $=90^{\circ}$ ). Each image consisted of 18 contiguous, $7-\mathrm{mm}$ thick axial slices acquired parallel to the anterior-posterior commissure plane $(3.75 \times 3.75 \mathrm{~mm}$ in-plane $)$, allowing complete brain coverage at a high signal-to-noise ratio (Conturo et al., 1996).

A total of 9 scanning runs were performed (3 runs of each condition), with each run consisting of two task blocks of 20 trials (94 scans), alternating with three fixation blocks that each lasted $37.5 \mathrm{sec}$ (15 scans). During task blocks, the intertrial interval (ITI) was variable, from $2.5 \mathrm{sec}$ to $7.5 \mathrm{sec}$ in units of $2.5 \mathrm{sec}$, in order to create the necessary temporal jitter to ensure robust deconvolution of event-related fMRI responses. There were 237 scans per run, with each run lasting approximately $10 \mathrm{~min}$. A 2-min delay, during which participants rested, occurred between runs. The first four images in each scanning run were used to allow the scanner to reach steady state, so were discarded.

Visual stimuli were presented using PsyScope software (Cohen, MacWhinney, Flatt, \& Provost, 1993) running on an Apple PowerMac G4. Stimuli were projected to participants with an AmPro LCD projector (Model 150) onto a screen positioned at the head end of the bore. Participants viewed the screen through a mirror attached to the head coil. A fiber optic, light-sensitive keypress interfaced with the PsyScope button box was used to record participants' behavioral performance.

\section{Data Analysis}

Behavioral data were analyzed to examine the effects of reward motivation on performance. Error rates and RTs were examined through a repeated measures ANOVA, followed by pairwise $t$ tests for significant effects. Two sets of analyses were conducted, a first stage examining global performance, and a second stage examining specific trial-type effects (using trial-type $\times$ condition factorial ANOVAs).

Functional imaging data were preprocessed prior to statistical analysis according to the following procedures: All functional images were first temporally aligned across the brain volume, corrected for movement using a rigid-body rotation and translation correction (Friston, Williams, Howard, Frackowiak, \& Turner, 1996; Snyder, 1996), and then registered to the participant's anatomical images in order to correct for movement between the anatomical and function scans. The data were then scaled to achieve a whole-brain mode value (used in place of mean because of its reduced sensitivity to variation in brain margin definition) of 1,000 for each scanning run (to reduce the effect of scanner drift or instability), resampled into 3-mm isotropic voxels and spatially smoothed with a 9-mm FWHM Gaussian kernel. Participants' structural images were transformed into standardized atlas space (Talairach \& Tournoux, 1988) using a 12-dimensional affine transformation (Woods, Cherry, \& Mazziotta, 1992; Woods, Grafton, Holmes, Cherry, \& Mazziotta, 1998). The functional images were then registered to the reference brain, using the alignment parameters derived for the structural scans.

A general linear model (Friston, Frith, Frackowiak, \& Turner, 1995) was used to estimate parameter values for both event-related responses (item effects) and for sustained activity associated with the entire task block (state effects). State effects can be independently coded into the GLM with an assumed response shape of long duration (a boxcar convolved with a gamma function). The logic of this approach is that event-related effects should decay back to baseline during the ITI, whereas the state effects should remain 
fairly constant. Event-related effects were analyzed separately for each trial type by estimating values for the time points within the hemodynamic response epoch, which was estimated to be $25 \mathrm{sec}$ (10 scanning frames). These time points were estimated using unassumed hemodynamic response shapes.

Imaging data were analyzed to identify regions showing selective increases in sustained activity associated with reward motivation. Regions showing this pattern had to satisfy a set of simultaneous contrasts: (1) significant sustained activation (relative to fixation) in the reward condition; (2) significantly greater sustained activity in reward relative to baseline; and (3) significantly greater sustained activity in reward relative to penalty. This last contrast ensured that the increased sustained activity in reward was not due to a nonselective increase in arousal or other strategic changes in processing resulting from either addition of financial incentives or the inclusion of no-go trials. All voxels within the region were required to be statistically significant in each of the three aforementioned contrasts ( $p<.05$, uncorrected). Moreover, a region was considered significant only if it contained a cluster of 8 or more contiguous voxels.

This approach — of using multiple simultaneous contrasts, with each set at a relatively low threshold - optimizes the trade-off between sensitivity/power and false-positive protection (i.e., Type I vs. Type II error). It is important to note that conjunction tests of this type do not provide enhanced false-positive protection against the hypothesis that at least one of the contrasts represents a false positive (Nichols, Brett, Andersson, Wager, \& Poline, 2005). However, according to the analysis provided by Friston, Penny, and Glaser (2005), one can validly use such analyses to test the inference that at least two of the three contrasts represent true positives. For such an inference, the voxelwise false positive rate is approximately .007 - although this may still be an overestimate, given nonindependence of the error terms in the contrasts. In addition, an 8-voxel cluster size requirement was used to increase protection against false positives (Forman et al., 1995; McAvoy, Ollinger, \& Buckner, 2001).

To further constrain the interpretation of the identified regions, we conducted two overlap analyses. First, we identified voxels showing significant trial-locked activation via an event-related analysis within the baseline condition. Voxels were identified that showed a significant event-related response through an ANOVA main effect of time within the 10-point GLM estimate of the trial response epoch. The map was thresholded at $p<.05$ corrected $(z=4.0$, cluster size $=7$; via a Monte Carlo procedure for estimating voxelwise significance and cluster-size; McAvoy et al., 2001). Second, we constructed an a priori functional ROI map of regions reliably associated with cognitive control and working memory. This map was generated by constructing spherical regions of $16-\mathrm{mm}$ radius centered on seed-point coordinates taken from two published metaanalyses (Owen, McMillan, Laird, \& Bullmore, 2005; Wager \& Smith, 2003).

Each of these two maps was then tested for overlap with the ROIs, identified to show an increase in sustained activation in relationship to reward incentive (via an AND operation). Regions of significant overlap (minimum 8 voxels) with the event-related response map and/or the canonical working memory/cognitive control map are noted in Table 1.

Next, by comparing the reward effect on sustained activity with the reward effect on event-related responses, the selectivity of the reward effects to sustained activation changes was also examined. This analysis was conducted by first computing the magnitude of the event-related response during Time Points 3-6, encompassing the cue, delay, and probe; and then by conducting a repeated measures ANOVA with incentive condition (baseline, reward) $\times$ activation dynamics (sustained $\times$ event-related) as factors. Selectivity to sustained effects was demonstrated through the presence of significant interaction. Two further analyses were conducted to see whether there were other regions with both high sustained and high event-related activity, and to compare the selectivity of the reward regions with respect to penalty.
A separate set of analyses was conducted to examine how individual differences modulated the effect of the motivational incentive manipulation. The first analysis examined individual differences related to behavioral performance and identified regions in which the level of sustained activity during the reward incentive condition was correlated with participants' rate of reward-specifically, the number of correct trials faster than their median RT divided by the total number of trials in the reward block. It is important to note that the reward rate measure takes into account individual variation in both RT and errors in the reward condition, since the reward criteria require that trials be both fast and correct to be rewarded. Moreover, this measure controls for individual differences in baseline performance, since the cutoff is directly and individually set according to each participant's baseline RT and error rate, in order to achieve an expected value of $50 \%$ reward rate, assuming no change in performance in the reward condition. Thus, variability in reward rate represents individual variation in the reward-induced change in performance from baseline.

The second analysis examined individual differences related to personality measures. This analysis identified regions in which the level of sustained activity during the reward incentive condition was correlated with a personality measure. Separate analyses were conducted testing for correlations with GRAPES and BAS measures. In all individual difference analyses, voxelwise correlation maps were computed but masked to include only voxels for which there was positive sustained activation on average across participants. This masking ensured that we would not identify regions that tended to be deactivated in the reward condition. Moreover, because of the low statistical power of our sample and the exploratory nature of these analyses, we used a false positive rate of $p<.05$, uncorrected. Thus, the results have an increased vulnerability to false positives, and as such should be considered tentative.

\section{RESULTS}

\section{Behavioral Results}

We first examined whether the incentive manipulation was successful at changing behavior at a global level. Initially, only AX trials were considered because they were the most frequent go trials; and as they were target trials, they were free from interference effects. Incentive effects were significant for both RTs $[F(2,30)=41.67$, $p<.001]$ and error rates $[F(2,30)=3.11, p=.059]$. The reward condition $(420 \mathrm{msec})$ was significantly faster than either baseline [571 msec; $t(15)=9.46, p<.001$ ] or penalty conditions [628 msec; $t(15)=7.84, p<.001]$. This speed-up in RT was striking, representing an over $25 \%$ improvement in performance from baseline (over $30 \%$ from penalty). Next, we looked to see if there was any difference in RT between participants who received the reward condition rather than the penalty condition first. We did find order-of-presentation effects, but only for the penalty condition. Participants who had penalty before reward $(M=728 \mathrm{msec})$ were significantly slower than were those participants who had penalty after reward $[M=562 \mathrm{msec} ; t(14)=2.35, p<.05]$. For reward, there was no significant difference between participants who had penalty before they had reward $(M=448 \mathrm{msec})$ and participants who had reward first $[M=422 \mathrm{msec}$; $t(14)=0.79$, n.s.].

The speeding of RT in reward did not come at the cost of increased errors, as the difference between reward $(3.7 \%$ errors) and baseline (1.8\%) condition was not significant $[t(15)=1.69$, n.s. $]$, nor was the difference between reward 
Table 1

Sustained Activity Effects of Reward-Related Motivation

\begin{tabular}{|c|c|c|c|c|c|c|c|}
\hline Brain Region & BA & $\begin{array}{c}\text { Size } \\
\left(\mathrm{mm}^{3}\right)\end{array}$ & $x$ & $y$ & $z$ & $\begin{array}{l}\text { Magnitude } \\
\text { (\% change) }\end{array}$ & $\begin{array}{c}\text { Peak } \\
z \text { Score }\end{array}$ \\
\hline \multicolumn{8}{|c|}{ Frontal Lobe } \\
\hline Right ventrolateral PFC ${ }^{*}$ & 45 & 216 & 46 & 21 & 18 & 0.089 & 2.35 \\
\hline Right midlateral $\mathrm{PFC}^{* \dagger}$ & 9 & 270 & 34 & 27 & 27 & 0.1143 & 1.91 \\
\hline Right IFJ/premotor cortex ${ }^{* \dagger}$ & 6 & 2,592 & 32 & 6 & 48 & 0.152 & 2.52 \\
\hline Dorsal anterior cingulate cortex ${ }^{* \dagger}$ & 32 & 297 & -16 & 6 & 39 & 0.0659 & 2.76 \\
\hline Presupplementary motor area ${ }^{* \dagger}$ & 6 & 1,836 & 10 & 6 & 51 & 0.1421 & 2.88 \\
\hline Right superior PFC & 8 & 405 & 20 & 30 & 48 & 0.107 & 2.36 \\
\hline Right supplementary motor area ${ }^{* \dagger}$ & 6 & 837 & 20 & -3 & 66 & 0.3042 & 2.64 \\
\hline Left premotor cortex ${ }^{*}$ & 4 & 486 & -20 & -24 & 66 & 0.2354 & 2.42 \\
\hline Right premotor area* & 6 & 378 & 2 & -18 & 75 & 0.4435 & 2.91 \\
\hline Right precentral gyrus* $*$ & 6 & 405 & 50 & 0 & 45 & 0.2467 & 2.49 \\
\hline \multicolumn{8}{|c|}{ Parietal Lobe } \\
\hline Right inferior parietal cortex* & 40 & 459 & 56 & -39 & 45 & 0.1967 & 2.37 \\
\hline \multicolumn{8}{|c|}{ Temporal Lobe } \\
\hline Right inferior temporal gyrus* & 20 & 324 & 56 & -18 & -33 & 0.5165 & 2.98 \\
\hline Left inferior temporal gyrus & 20 & 540 & -46 & -30 & -27 & 0.2966 & 2.65 \\
\hline Left fusiform gyrus $* \dagger$ & 37 & 513 & -50 & -51 & -15 & 0.2771 & 2.52 \\
\hline Left parahippocampal gyrus* & 28 & 540 & -20 & -27 & -6 & 0.1593 & 2.41 \\
\hline \multicolumn{8}{|c|}{ Cerebellum } \\
\hline Left inferior posterior cerebellum ${ }^{* \dagger}$ & & 540 & -28 & -78 & -45 & 0.3682 & 4.07 \\
\hline Left inferior lateral cerebellum & & 405 & -46 & -60 & -42 & 0.3011 & 2.47 \\
\hline Left cerebellum* $* \dagger$ & & 2,538 & -32 & -63 & -33 & 0.1964 & 3.00 \\
\hline Left medial cerebellum* $*$ & & 945 & -10 & -72 & -33 & 0.1225 & 3.14 \\
\hline Left lateral posterior cerebellum & & 837 & -40 & -78 & -30 & 0.6376 & 2.97 \\
\hline \multicolumn{8}{|c|}{ Subcortical } \\
\hline Pons* & & 1,188 & -2 & -36 & -21 & 0.1601 & 2.50 \\
\hline
\end{tabular}

Note-Regions showing sustained activation related to reward in terms of Brodmann's area (BA), center of mass in Talairach coordinates. Magnitude of percentage signal change and peak $z$ value refer to the reward condition. Regions whose center-of-mass coordinates are outside the brain per the Talairach but appear to be within the brain in the image files are listed in italics. Bolded regions exhibited a significant condition (baseline vs. reward) by effect type (item vs. state) interaction, in the selectivity analysis. Regions marked with an * exhibited a significant main effect of time in the baseline block. Regions marked with a ${ }^{\dagger}$ overlap with regions reliably associated with working memory and cognitive control in meta-analyses (Owen et al., 2005; Wager \& Smith, 2003). PFC, prefrontal cortex; IFJ, inferior frontal junction.

and penalty $[4.5 \%$ errors, $t(15)=0.66$, n.s.]. The main effect of incentive was instead due to increased errors in penalty relative to baseline $[t(15)=2.98, p<.01]$.

These results indicate that the reward manipulation was highly successful in altering participants' performance. The penalty condition was also successful in altering participants' performance, since the incentive to avoid no-go errors during this condition resulted in significantly fewer such errors than in the reward condition [penalty $=1.6 \%$, reward $=20.6 \% ; t(15)=5.024, p<.001]$. Additionally, in the penalty condition, significantly fewer no-go errors were made than go errors $[$ no-go $=1.6 \%$, go $=4.6 \%$; $t(15)=4.73, p<.001]$, though the opposite was true in reward (no go $=20.6 \%$, go $=4.5 \%$ ), yielding a significant condition $\times$ trial type interaction $[F(1,15)=24.23$, $p<.001]$.

We next examined performance effects broken down by trial type, in order to reveal any more specific effects of incentive condition on task strategies (see Table 2 for a summary of results). Repeated measures ANOVAs were conducted with within-subjects factors of condition (baseline, reward, penalty) and trial type (AX, AY, BX, BY). Significant condition $X$ trial type interactions were observed for both errors $[F(6,90)=3.65, p<.01]$ and $\mathrm{RT}[F(6,90)=$
$6.45, p<.001]$. These were followed by computing measures of AY and BX interference effects (expressed by subtracting performance in the BY control condition) for both $\mathrm{RT}$ and error measures. As predicted, in terms of errors AY interference was significantly increased in the reward condition $(10.6 \%)$ relative to both the baseline $[0.6 \% ; t(15)=$ $3.56, p<.01]$ and penalty $[1.2 \% ; t(15)=3.44, p<.01]$ conditions. There was no significant AY interference for RT. For BX trials, RT interference was significantly reduced in the reward condition $(15 \mathrm{msec})$ relative to penalty $[102 \mathrm{msec} ; t(15)=4.98, p<.001]$ and was equivalent to baseline [13 msec, $t(15)=0.097$, n.s.]. There was no significant BX interference for errors. These results support the hypothesis that participants adopted a proactive control strategy in the reward condition, since such a strategy would generally reduce BX interference while increasing interference on AY trials.

Lastly, we examined individual difference effects. The dramatic reward-related enhancement of RT led to higher rates of obtained reward than expected from the baseline condition. The average rate of reward during the reward block was $81 \%$, which was over $30 \%$ higher than the expected rate of approximately $50 \%$ reward set from the baseline reward criterion. However, even though all par- 
Table 2

Reaction Times (RTs, in Milliseconds) and Error Rates by Trial Type and Condition

\begin{tabular}{|c|c|c|c|}
\hline \multirow[b]{2}{*}{ Trial Type } & \multicolumn{3}{|c|}{ Condition } \\
\hline & Baseline & Reward & Penalty \\
\hline \multicolumn{4}{|c|}{ RTs } \\
\hline $\mathrm{AX}$ & 570.73 & $420.36^{* * *}$ & $628.21^{*}$ \\
\hline $\mathrm{AY}$ & 714.54 & $554.28^{* * *}$ & 726.23 \\
\hline $\mathrm{BX}$ & 581.48 & $426.66^{* * *}$ & $701.48^{*}$ \\
\hline BY & 568.33 & $411.43^{* * *}$ & 598.97 \\
\hline \multicolumn{4}{|c|}{ Error Rates } \\
\hline $\mathrm{AX}$ & .018 & .037 & $.045^{* *}$ \\
\hline AY & .017 & $.111^{* *}$ & .033 \\
\hline $\mathrm{BX}$ & .056 & .057 & .08 \\
\hline BY & .01 & .005 & .021 \\
\hline No-go & - & .206 & $.016^{* * *}$ \\
\hline
\end{tabular}

Note-For RTs, asterisks indicate a significant difference from baseline. For error rates, asterisks indicate significant difference from baseline for go trials, and from reward for no-go trials. ${ }^{*} p<.05 .{ }^{* *} p<$ .01 . ${ }^{* * *} p<.001$

ticipants showed an increase in expected reward rate, there was also a high degree of variability across participants, with a range of obtained rewards from $64 \%$ to $98 \%$ of trials. This suggests that participants were affected in varying ways by the reward incentive manipulation. We next examined whether the two personality variables BAS and GRAPES could explain any of this variability. Whereas GRAPES was not correlated with percent reward $(r=$ -.042 , n.s.), BAS showed a marginally significant correlation with percent reward $(r=.47, p=.07)$. This positive correlation suggests that examination of the neural basis of personality and performance-based variability might be a profitable approach.

\section{Imaging Results - Sustained Activity}

We identified regions showing sustained activation selectively in the reward incentive condition. This analysis revealed prominent activation in a number of right lateralized dorsal, ventral, and posterior PFC and motor regions, along with activity in additional regions such as the right parietal cortex, the dorsal medial frontal cortex (i.e., anterior cingulate cortex and presupplementary motor area) and the cerebellum (see Table 1 and Figure 1).

Given the exploratory nature of this analysis, we also investigated the extent to which these regions corresponded with regions that had a significant event-related response during task performance in the baseline AX-CPT condition, or were reliably involved during working memory and cognitive control, as assessed in two recent meta-analyses by Owen et al. (2005) and Wager and Smith (2003). The correspondence was assessed via an explicit overlap analysis. The results demonstrated that the majority of regions (17/21) showed significant task-related activity in the form of an event-related response during baseline. Many regions $(10 / 21)$ also overlapped in anatomic localization with regions found to be reliably associated with working memory and cognitive control. The fact that these overlapping regions included the anterior and posterior lateral PFC, the dorsal medial frontal cortex, and the cerebellum supports the idea that brain regions engaged by baseline AX-
CPT performance, and typically associated with working memory and cognitive control functions, also demonstrate sensitivity to reward incentive motivational states.

\section{Selectivity Analysis}

We next examined whether the reward-related enhancement in activity in these regions was selective to sustained responses. To test this issue, the magnitude of the reward incentive effect on event-related responses was compared with the effect on sustained response via a condition $\times$ activation dynamics repeated measures ANOVA conducted on the ROIs identified in the previous analysis. Almost all regions (19/21) showed a significant interaction, with the remaining regions showing a marginal interaction. Moreover, in no region was there a significant increase in event-related activity during the reward condition. In fact, the form of the interaction was almost always a crossover pattern in which event-related activity tended to decrease in the reward condition (albeit nonsignificantly), whereas sustained activity increased (see Figure 2 for a graph of activity in the right inferior frontal junction).

Looking for regions with high sustained activity, then looking at event-related activity in those regions, may present a biased picture, given that regions with the highest sustained activity would probably not show as high or higher event-related activity. To address this concern, we investigated whether there were regions that we had not considered that showed both state and item activity in reward. A conjunction analysis looking for reward item activity and reward state activity significantly greater than fixation revealed several parietal and posterior frontal regions. However, these regions were no longer significant when constrained to be significantly greater than baseline; this suggested that the activation was not selective to the incentive manipulation. A second follow-up analysis, looking at selectivity with respect to penalty rather than baseline, found similar results. All regions maintained a crossover pattern, although only $11 / 21$ showed a significant interaction. These results demonstrate that the reward-related motivational context produced a sustained increase in brain activation that was relatively selective, in that it occurred in a network of cognitive control-related regions, even independently of any event-related changes in this network.

Alternative interpretations. An alternative interpretation of the activity in these regions is that they reflect a greater inhibitory demand in reward than they do in baseline (because baseline did not include no-go trials) or in penalty (because there was less pressure to perform quickly in penalty). To look into this possibility, we examined the reward effect on event-related responses for go trials (separate analyses were done both on AX targets and by averaging all types together) and no-go trials separately, in the previously identified sustained ROIs. If activation in these ROIs primarily reflected changes in inhibitory demand in these regions, we should have predicted greater event-related responses on the no-go inhibitory trials relative to go trials, as had been observed in many previous studies (e.g., Konishi et al., 1999; Liddle, Kiehl, \& Smith, 2001). However, this pattern was not evident in the major- 


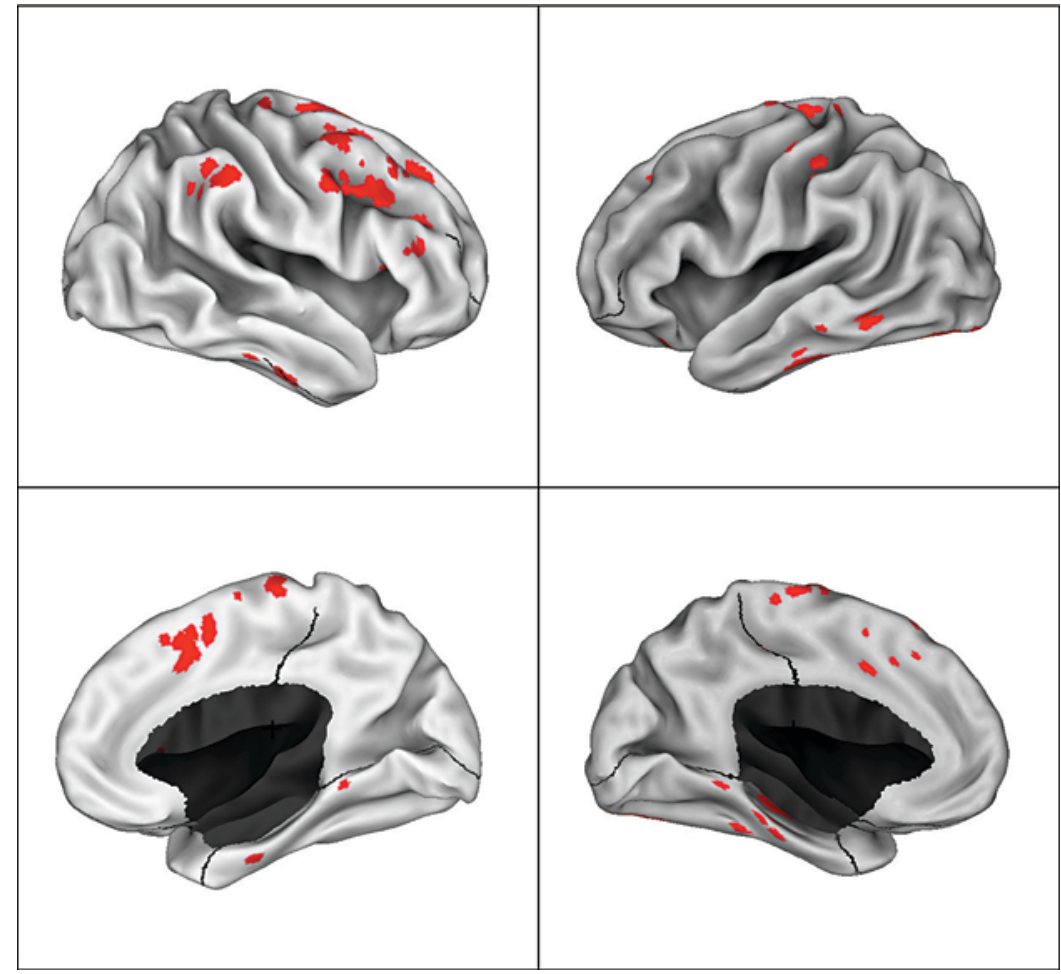

Figure 1. Regions highlighted show sustained activity in the reward condition that is significantly greater than the fixation, baseline, and penalty conditions.

ity of regions. Only 4 of 22 regions showed the general numerical pattern of reward no-go $>$ reward go; moreover, in none of these regions was the effect significant.

A second prediction of this alternative account is that the greater inhibitory demands of the reward condition relative to penalty would be reflected in terms of increased event-related responses on no-go trials in reward versus penalty. However, none of the 21 regions showed penalty activity greater than reward activity for these trial types.
Figure 3 shows the pattern of event-related responses across task conditions in a representative sustained ROI from the anterior cingulate cortex. It is important to note that the failure to find support for these predictions within regions identified in our original analysis does not rule out the possibility that these regions do contribute to inhibitory processes. Rather, it only indicates that they are likely not involved in the type of inhibition that can be indexed by event-related activation on no-go trials in this task.

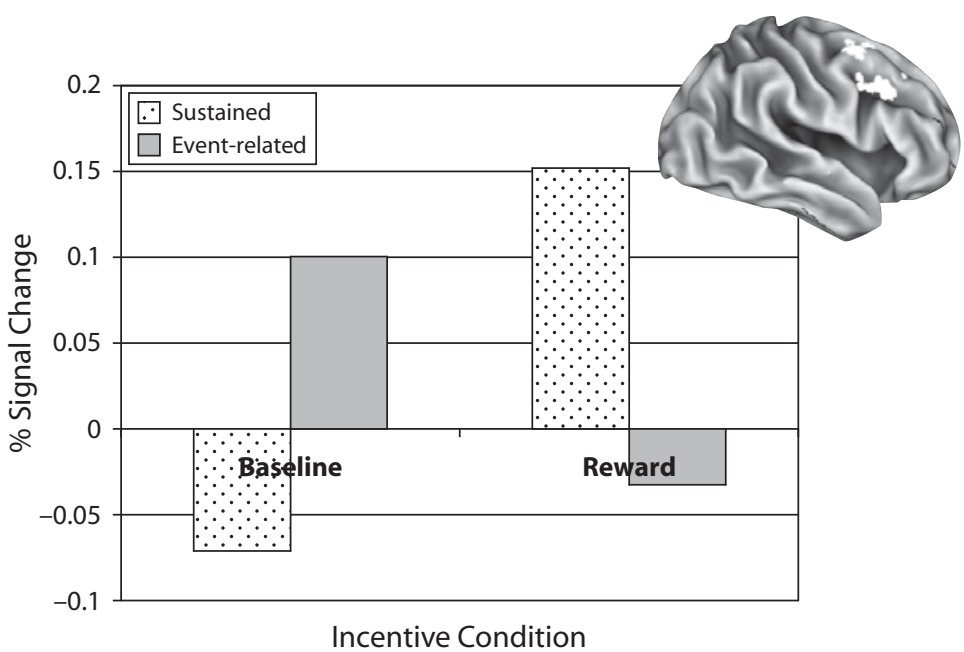

Figure 2. Activity in right inferior frontal junction, showing an incentive condition by effect type (sustained vs. event-related) crossover interaction. 


\section{Analyses of Individual Differences}

A second set of voxelwise whole-brain analyses examined correlations with sustained activity in the reward condition. Because of the low statistical power and exploratory nature of these analyses, they should be interpreted tentatively and replicated in future studies. Nevertheless, we report them here, since - to our knowledge - they represent the first data on the effects of individual differences on motivation-related brain activation. The full results are reported primarily for archival purposes, and we focus only on activations in brain regions that might be expected from prior work, such as the lateral PFC and regions thought to be associated with reward processing.

Performance. We identified regions showing sustained (positive) activation that was significantly positively correlated with the reward-related performance effect (i.e., individual differences in reward rate). Active regions included the frontopolar cortex (see Table 3 and Figure 4). Interestingly, none of the regions identified in this analysis overlapped with the regions identified in the group averaged sustained analysis. To provide converging evidence for this point, we masked the sustained activity analysis map with a performance analysis correlation map to see whether any sustained voxels also showed a significant relationship to performance variability. Only a few isolated voxels showed any such performance effects, confirming that the two analyses identified nonoverlapping sets of regions.

Personality. Next, correlation analyses were conducted that examined whether state activity in the reward condition was associated with scores on the GRAPES reward subscale and on the BAS scale, two personality measures theoretically related to reward. Significant correlations were observed in regions linked to reward processing and higher level components of cognitive control, and these were followed with focused ROI analyses. The GRAPES analysis identified activation in the subcallosal gyrus, whereas the BAS analysis identified activation in the $\mathrm{OFC}$, the caudate nucleus, and the frontopolar PFC (Figure 4).

None of these regions showed significant correlations between baseline or penalty activity and personality. In addition, the magnitude of the correlation between personality and sustained activity in the reward condition was significantly greater than the magnitude of the correlation observed in the baseline and penalty conditions in the subcallosal gyrus (baseline, $z=3.09, p<.01$; penalty, $z=$ 4.24, $p<.0001$ ) and frontopolar cortex (baseline, $z=$ $3.02, p<.01$; penalty, $z=2.55, p<.05$ ), as determined by Meng, Rosenthal, and Rubin's (1992) procedure for comparing correlated correlation coefficients. The magnitude of the reward correlation was only marginally greater than in the baseline and penalty conditions in the caudate nucleus (baseline, $z=1.89, p=.059$; penalty, $z=1.91$, $p=.056$ ), and the reward correlation in OFC was significantly greater than penalty $(z=4.89, p<.001)$ but not baseline $(z=1.39$, n.s. $)$.

Like the performance analysis, the personality analysis appeared to identify a distinct set of regions from that found in the sustained and performance-based analyses. We tested this by masking the sustained activity map with the two personality maps. Again, only a few isolated voxels showed both significant sustained activity and personality-related variation, confirming that the two analyses identified nonoverlapping sets of regions.

\section{DISCUSSION}

The aim of the present study was to investigate the impact of reward and motivation on brain activity and be-

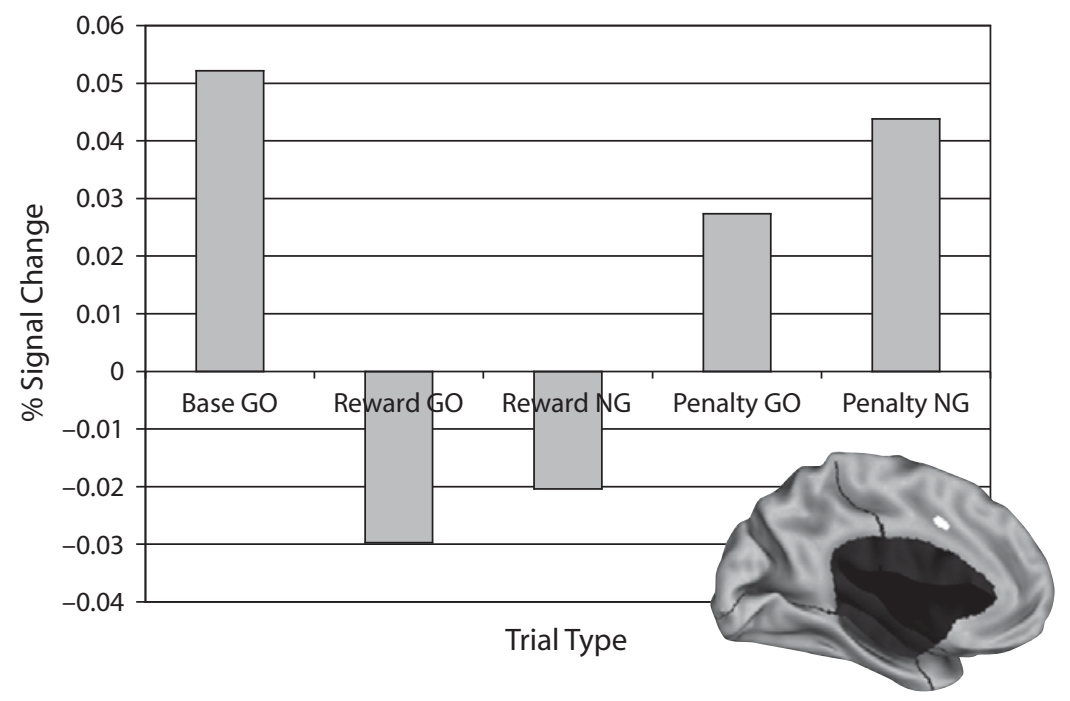

Figure 3. Event-related activity across task conditions for both go and no-go trials, shown for a representative region in dorsal anterior cingulate cortex. The deactivation of no-go responses in the reward condition and increased activation in the penalty condition is not consistent with a hypothesis of increased inhibitory demands in the reward condition. 
Table 3

Individual Difference Effects on Sustained Activity

\begin{tabular}{|c|c|c|c|c|c|c|}
\hline & BA & $\begin{array}{c}\text { Size } \\
\left(\mathrm{in} \mathrm{mm}^{3}\right)\end{array}$ & $x$ & $y$ & $z$ & $\begin{array}{c}\text { Pearson } \\
\quad R\end{array}$ \\
\hline \multicolumn{7}{|c|}{ Proportion Rewarded Trials } \\
\hline Left frontopolar cortex & 10 & 216 & -32 & 60 & 6 & .52 \\
\hline Right frontopolar cortex & 10 & 243 & 20 & 61 & -2 & .49 \\
\hline Left inferior frontal gyrus & 47 & 243 & -50 & 40 & -7 & .56 \\
\hline Right inferior parietal cortex & 40 & 243 & 52 & -66 & 40 & .54 \\
\hline Left middle temporal cortex & 21 & 270 & -62 & -26 & -4 & .51 \\
\hline Right middle temporal cortex & 21 & 297 & 65 & -19 & -11 & .51 \\
\hline Right parahippocampal gyrus & 36 & 216 & 18 & -8 & -35 & .56 \\
\hline Left inferior cerebellum & & 675 & -20 & -51 & -51 & .59 \\
\hline Right medial cerebellum & & 378 & 23 & -55 & -45 & .52 \\
\hline Left lateral cerebellum & & 540 & -53 & -62 & -32 & .66 \\
\hline Pons & & 2,457 & 3 & -26 & -42 & .62 \\
\hline Pons & & 216 & -14 & -21 & -35 & .55 \\
\hline \multicolumn{7}{|c|}{ GRAPES } \\
\hline Subcallosal gyrus & & 567 & 14 & 9 & -12 & .63 \\
\hline Left cerebellum & & 405 & -46 & -42 & -30 & .58 \\
\hline Left brainstem & & 702 & -14 & -33 & -42 & .57 \\
\hline \multicolumn{7}{|c|}{ BAS } \\
\hline Right frontopolar cortex & 10 & 216 & 32 & 54 & 21 & .49 \\
\hline Right superior PFC & 8 & 540 & 10 & 42 & 36 & .52 \\
\hline Bilateral OFC & 11 & 1,188 & 2 & 21 & -24 & .64 \\
\hline Right inferior temporal gyrus & 20 & 324 & 56 & -30 & -18 & .55 \\
\hline Right caudate nucleus & & 972 & 2 & 15 & -6 & .58 \\
\hline Left inferior cerebellum & & 3,051 & -26 & -45 & -45 & .68 \\
\hline Left lateral cerebellum & & 378 & -46 & -63 & -36 & .59 \\
\hline Left posterior cerebellum & & 783 & -34 & -84 & -30 & .55 \\
\hline Left brainstem region & & 324 & -10 & -27 & -45 & .55 \\
\hline
\end{tabular}

Note-Regions showing correlation of individual differences with sustained activation in the reward condition, in terms of Brodmann's area (BA), center of mass in Talairach coordinates. Peak $R$ value refers to the correlation effect size with the relevant individual difference variable. PFC, prefrontal cortex; OFC, orbital frontal cortex.

havior. Results of the imaging analyses indicated that the reward incentive context was associated with an increased level of tonic activity in a network of brain regions separable from changes in transient, trial-by-trial activity. Both the regions that were activated, as well as the results of behavioral analysis on the pattern of RTs and error rates, suggest that the effect of the incentives was primarily on cognitive control processes. In addition to the observed group effects, there were indications that activity in some regions was variable across individuals, reflecting differences in performance and personality. Both of these points will be treated in greater detail below.

\section{Motivation and Cognitive Control}

Behavioral results showed a striking speed-up of RTs in the reward condition, without a concomitant increase in errors (in AX trials, which were the most frequent). This means that in the majority of trials participants were successful at responding faster while maintaining accuracy. Importantly, the increase in RT speed was observed for all trial types, whereas we observed a significant increase in error rates for only one trial type, $\mathrm{AY}$. The fact that $\mathrm{AY}$ trials were more error-prone supports our prediction that the reward condition would be associated with a proactive control strategy, whereby context (the cue) is actively maintained. The AY trial type is the only one that should be adversely affected by proactive control, because the strong A-cue representation strongly primes the target response, given that $87.5 \%$ of A-cues are followed by an $X$ probe. The use of proactive control is also potentially a more cognitively effortful strategy, in that it requires active maintenance of contextual expectancies during delay periods (and even potentially across trials). Thus, a proactive cognitive control strategy may only be strongly preferred in task situations that offset both the adverse effects and the generalized effort costs. The reward incentive condition may represent a situation like this that favors the use of proactive control.

Regions identified in the imaging analysis also support the interpretation of a possible shift in cognitive control strategy. An increase in sustained activity during the reward block was found in a network of regions including the right lateral PFC (RLPFC - including superior, dorsolateral, ventrolateral, and posterior regions, such as the inferior frontal junction, or IFJ), the right parietal cortex, the dorsal medial frontal cortex (including the anterior cingulate), and the left cerebellum. This network appears to match well with the network typically engaged by working memory and cognitive control tasks, as directly verified by an overlap analysis with ROIs obtained from meta-analyses (Owen et al., 2005; Wager \& Smith, 2003). The components of this network may reflect specialized control processes affected by shifts in incentive state.

RLPFC and right parietal cortex are thought to be the core components of a neural circuit that mediates sus- 


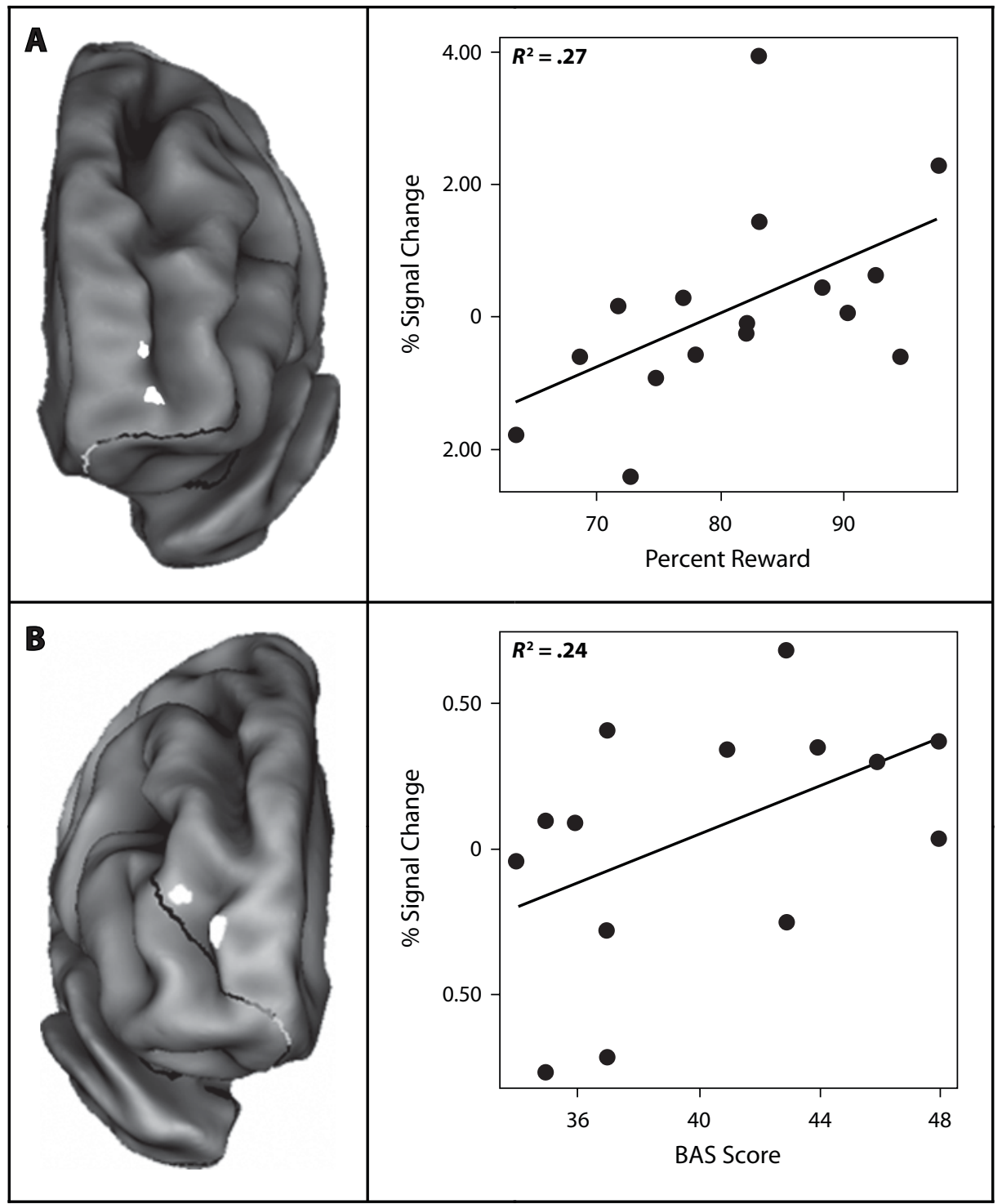

Figure 4. Regions showing significant positive correlations with individual difference variables. (A) Left frontopolar cortex region showing correlation between reward state activity and percent reward. (B) Right frontopolar cortex region showing correlation between reward state activity and BAS scores.

tained attention (Pardo et al., 1991; Posner \& Petersen, 1990). During long and repetitive tasks, it may be tempting to let attention wander. Having high state activity in RLPFC and right parietal cortex in the reward condition may enable participants to avoid being distracted, and therefore make faster and more correct responses, to gain more reward.

A specific region within the posterior RLPFC that showed increased tonic activity included the IFJ. The IFJ has been shown to be active across a variety of cognitive tasks, including task switching, working memory, and response inhibition tasks (Derrfuss, Brass, \& von Cramon, 2004). Derrfuss, Brass, Neumann, and von Cramon (2005) confirmed this result with a meta-analysis involving a range of tasks, concluding that the IFJ should be considered a functionally distinct component of the cognitive control system, specifically as a mediator of task preparation. Being prepared for each trial by having an active representation of the task could help in responding faster and more accurately to stimuli, ultimately increasing rewards.

Right ventrolateral frontal cortex (RVLPFC) has been implicated in cognitive inhibition, or the suppression of irrelevant thoughts, memories, or responses (Aron, Robbins, \& Poldrack, 2004). For example, activity in RIFC has been associated with the successful inhibition of a prepotent response in a stop task (Rubia, Smith, Brammer, \& Taylor, 2003). The subregion of RVLPFC found in the present study, BA 45, may have played a similar role in participants' performance in the AX-CPT task. Aside 
from the no-go trials, all trials require a response, and for most of the trials $(70 \%)$ the correct response is the target response. Thus, when presented with a nontarget trial (AY, $\mathrm{BX}$, or BY), it is necessary to inhibit the dominant tendency to make a target response. A high state level of activity may have facilitated this inhibition, leading to more correct responses.

The medial frontal cortex, particularly the dorsal anterior cingulate, has been implicated in performance monitoring functions. According to a computational model by Botvinick, Braver, Barch, Carter, and Cohen (2001), due to activation of competing responses, the anterior cingulate is engaged by conflict. The detection of conflict in the anterior cingulate in turn recruits higher control structures, presumably within the lateral PFC. The sustained activity in the present study, then, may indicate that reward brought about increased performance monitoring. If participants were experiencing conflict about whether to choose the target or nontarget responses, better cognitive control at higher levels - for example, to more strongly inhibit interference - might help to resolve that conflict.

Several regions within the left cerebellum also showed tonically increased activity under reward conditions. This activity may be reflective of the cerebellum's hypothesized role as a contributor to verbal working memory. Specifically, it is thought that the cerebellum may act as an error detection system during verbal rehearsal, in much the same way as it detects errors in the execution of complex motor plans (Desmond \& Fiez, 1998; Fiez, 1996). Thus, increased cerebellar activity may reflect greater effort to maintain the accuracy of stimuli in working memory. Alternatively, the cerebellum has been associated with precise interval timing, for example, in tasks that require participants to estimate the duration of stimuli (Ivry, 1997; Ivry \& Spencer, 2004). Thus, the tonically increased activity in the cerebellum may represent more accurate timing of trial events, which may facilitate motor preparation. In the present study, participants who more accurately predicted the onset of the probe may have been more prepared to make a response, and thus may have been faster and more likely to obtain a reward.

Together, the increased tonic activity in these regions may reflect a greater level of cognitive control exerted by participants in the reward-incentive condition in order to improve performance and maximize reward attainment. Keeping active in a sustained rather than transient manner those regions that sustain attention, limit interference, and evaluate conflict may make for fewer lapses in control and lead to better performance. Comparing item activity and state activity in these state regions revealed that the increase associated with reward was selective to the sustained activity. In the baseline condition, these regions do tend to show a significant event-related response (again, assessed via an overlap analysis), and in fact, the eventrelated response was greater than was the sustained one. However, in the reward condition, this pattern reversed: Sustained activity was greater than the event-related response. This may suggest a shift in strategy from trial-bytrial initiation of control to sustained maintenance of control. Keeping control active in a tonic way may be a more effective - but potentially metabolically more demanding - way of preventing distraction and interference.

An alternative explanation is that these regions are active in a state-like manner, not because of the need to maintain control but because of the nature of the reward manipulation. Perhaps if reward were manipulated on a trial-by-trial basis rather than in a blocked way, these regions would show event-related activity rather than sustained activity. Although this argument cannot be dismissed outright, it seems more plausible that regions that are able to prevent interference and distraction would be active in a sustained manner in many kinds of incentive situations, because lapses in such control could lead to negative occurrences such as loss of potential reward or earning a penalty.

Although increased activity in these regions cannot be reduced to nonspecific effects of increased arousal or saliency because of the comparison with the penalty condition, the fact remains that we cannot strongly claim that the observed regions show selectivity to reward valence effects. For such claims to be made, a penalty condition tightly matched to the reward condition - that is, with penalties for slow and error trials based on equivalent performance criteria - would have to be included. Without such a condition, we cannot rule out the possibility that these same regions might be active when the incentives for performance are negative rather than positive. Indeed, Panksepp's theory of nucleus accumbens dopamine function, in which dopamine facilitates flexible approach toward rewards, can be equally applied to penalties: Avoiding a penalty entails an approach toward safety, an incentive (Ikemoto \& Panksepp, 1999). Thus, it seems surprising that no published studies, to our knowledge, have directly examined the motivational effects of tightly matched reward and penalty conditions on cognitive brain activity and task performance (but see Small et al., 2005, for an exception). This is an important direction for future work.

\section{Activity Related to Individual Differences}

Bilateral regions in the frontopolar cortex were identified in which sustained activity in the reward block was positively correlated with the rate of reward that participants achieved. The frontopolar cortex has been implicated in maintaining an overall goal while simultaneously keeping other goals in mind (Koechlin et al., 1999). Alternatively, the frontopolar cortex has also been implicated in subgoal processing, or taking a larger goal and breaking it down into the smaller goals needed to achieve it (Braver \& Bongiolatti, 2002). Gaining reward could be a fairly vague intention without being broken down into smaller parts that reflect elements of the task that can be improved. Thus, participants who were able to maintain an active representation of the high-level reward goal, and use this to appropriately bias more task-related subgoals, may have been able to more directly benefit from the reward incentives in terms of increased performance enhancement. Pochon et al. (2002) found frontopolar activity in two contexts: with increased working memory demand, and in response to reward independent of load. This pattern of data fits with ours, in that it suggests that, in keeping a personally relevant goal to gain 
reward active, while managing subgoals related directly to the cognitive task at hand, frontopolar PFC has both cognitive and affective roles to play.

Given that the aim of the present study was to produce a motivational state by offering rewards, personality variables that could have influenced participants' perceptions of rewards were measured and correlated with brain activity. Reward expectancy (GRAPES) and sensitivity to reward (BAS) were chosen to reflect individual differences in reward perception, with the idea that these might have demonstrable effects on brain activity. Both measures correlated with sustained activity in reward-related regions, including the subcallosal gyrus, the OFC, and the caudate nucleus. In addition, BAS was also correlated with activity in the right frontopolar cortex, in a region slightly overlapping the region correlated with percent reward.

It is interesting to note the very low overall magnitude of reward state activity across participants in the frontopolar cortex, the $\mathrm{OFC}$, and other regions related to individual difference measures. This suggests that traditional analyses that do not take individual differences into account may not have detected activity in these regions, even though they had quite high activity for high BAS or GRAPES participants. Traditional analyses reveal only what is common across participants by taking a group average, whereas individual differences analyses highlight regions that are especially variable in their activity. This makes individual difference analyses complementary to, rather than convergent with, traditional group analyses. Thus, they may highlight regions that do not mediate core processes, but rather represent the application of optional resources. This may be particularly relevant to motivation, since greater levels of motivation should be associated with increased use of resources to obtain desired rewards. These initial results are, nevertheless, tentative, due to the low statistical power and the potential for false positives in our analysis procedure. However, we report them in the hope of spurring further research into the effects of individual differences on the neural mechanisms of rewardrelated motivation and cognitive control.

\section{SUMMARY AND CONCLUSIONS}

This study aimed to look at the impact of motivation and individual differences on brain activity and behavior during a cognitive task. Of particular interest were those regions that maintained a sustained level of activity throughout the reward block. Sustained activity was primarily found in cognitive control regions, indicating that higher levels of motivation might act to increase cognitive control to sustain attention and prevent interference, thus maximizing reward. Individual differences in aspects of reward perception modulated sustained activity in rewardprocessing regions, and a region in the frontopolar cortex that may be involved in maintaining an overall goal of achieving reward. By and large, our results show that motivational incentives are associated with better performance and greater cognitive control, and that relationships between reward and brain activity may be modulated by how participants perceive reward.

\section{AUTHOR NOTE}

This work was supported by NIH Grant R01MH066078 (T.S.B.) and a Mr. and Mrs. Spencer T. Olin Fellowship for Women in Graduate Study (H.S.L.). The authors thank Christine Hoyer and Jessica Paxton for data collection and technical assistance, and the anonymous reviewers who provided helpful feedback on the manuscript. Correspondence concerning this article should be addressed to H. S. Locke, Department of Psychology, Washington University, Campus Box 1125, One Brookings Drive, St. Louis, MO 63130 (e-mail: hmsypher@artsci.wustl.edu).

Note-This article was accepted by the previous editorial team, when John Jonides was Editor.

\section{REFERENCES}

Aron, A. R., Robbins, T. W., \& Poldrack, R. A. (2004). Inhibition and the right inferior frontal cortex. Trends in Cognitive Sciences, $\mathbf{8}$, 170-177.

Ball, S., \& Zuckerman, M. (1990). Sensation seeking, Eysenck's personality dimensions and reinforcement sensitivity in concept formation. Personality \& Individual Differences, 11, 343-353.

Barch, D. M., Braver, T. S., Nystrom, L. E., Forman, S. D., Noll, D. C., \& Cohen, J. D. (1997). Dissociating working memory from task difficulty in human prefrontal cortex. Neuropsychologia, 35, 1373-1380.

Botvinick, M. M., Braver, T. S., Barch, D. M., Carter, C. S., \& Cohen, J. D. (2001). Conflict monitoring and cognitive control. Psychological Review, 108, 624-652.

Braver, T. S., Barch, D., \& CohEN, J. (1999). Cognition and control in schizophrenia: A computational model of dopamine and prefrontal function. Biological Psychiatry, 46, 312-328.

Braver, T. S., \& Bongiolatti, S. (2002). The role of frontopolar cortex in subgoal processing during working memory. NeuroImage, 15, 523-536.

Braver, T. S., Cohen, J. D., \& Barch, D. M. (2002). The role of the prefrontal cortex in normal and disordered cognitive control: A cognitive neuroscience perspective. In D. T. Stuss \& R. T. Knight (Eds.), Principles of frontal lobe function (pp. 428-447). New York: Oxford University Press.

Braver, T. S., Gray, J. R., \& Burgess, G. C. (2007). Explaining the many varieties of working memory variation: Dual mechanisms of cognitive control. In A. R. A. Conway, C. Jarrold, M. J. Kane, A. Miyake, \& J. N. Towse (Eds.), Variation in working memory (pp. 76-106). New York: Oxford University Press.

Braver, T. S., Reynolds, J. R., \& Donaldson, D. I. (2003). Neural mechanisms of transient and sustained cognitive control during task switching. Neuron, 39, 713-726.

Braver, T. S., Satpute, A. B., Rush, B. K., Racine, C. A., \& Barch, D. M. (2005). Context processing and context maintenance in healthy aging and early-stage dementia of the Alzheimer's type. Psychology \& Aging, 20, 33-46.

Breiter, H. C., \& Rosen, B. R. (1999). Functional magnetic resonance imaging of brain reward circuitry in the human. In J. F. McGinty (Ed.), Advancing from the ventral striatum to the extended amygdala: Implications for neuropsychiatry and drug abuse. In honor of Lennart Heimer (Annals of the New York Academy of Sciences, Vol. 877, pp. 523-547). New York: New York Academy of Sciences.

Carter, C. S., Braver, T. S., BarCh, D. M., Botvinick, M. M., Noll, D., \& CoHEN, J. D. (1998). Anterior cingulate cortex, error detection, and the online monitoring of performance. Science, 280, 747-749.

Carver, C. S., \& White, T. L. (1994). Behavioral inhibition, behavioral activation, and affective responses to impending reward and punishment: The BIS/BAS scales. Journal of Personality \& Social Psychology, 67, 319-333.

Cohen, J. D., MacWhinney, B., Flatt, M., \& Provost, J. (1993). PsyScope: An interactive graphic system for designing and controlling experiments in the psychology laboratory using Macintosh computers. Behavior Research Methods, Instruments, \& Computers, 25, 257-271.

Conturo, T., McKinstry, R., Akbudak, E., Snyder, A., Yang, T., \& RaICHLe, M. (1996). Sensitivity optimization and experimental design in functional magnetic resonance imaging. Society for Neuroscience Abstracts, 22, 7 . 
Delgado, M. R., Locke, H. M., Stenger, V. A., \& Fiez, J. A. (2003). Dorsal striatum responses to reward and punishment: Effects of valence and magnitude manipulations. Cognitive, Affective, \& Behavioral Neuroscience, 3, 27-38.

Delgado, M. R., Nystrom, L. E., Fissell, C., Noll, D. C., \& Fiez, J. A. (2000). Tracking the hemodynamic responses to reward and punishment in the striatum. Journal of Neurophysiology, 84, 3072-3077.

Derrfuss, J., Brass, M., NeumanN, J., \& von Cramon, D. Y. (2005). Involvement of the inferior frontal junction in cognitive control: Metaanalysis of switching and Stroop studies. Human Brain Mapping, 25, 22-34.

Derrfuss, J., Brass, M., \& von Cramon, D. Y. (2004). Cognitive control in the posterior frontolateral cortex: Evidence from common activations in task coordination, interference control, and working memory. Neurolmage, 23, 604-612.

Desmond, J. E., \& Fiez, J. A. (1998). Neuroimaging studies of the cerebellum: Language, learning, and memory. Trends in Cognitive Sciences, 2, 355-362.

Fiez, J. A. (1996). Cerebellar contributions to cognition. Neuron, 16, 13-15.

Forman, S. D., Cohen, J. D., Fitzgerald, M., Eddy, W. F., Mintun, M. A., \& NolL, D. C. (1995). Improved assessment of significant activation in functional magnetic resonance imaging (fMRI): Use of a cluster-size threshold. Magnetic Resonance in Medicine, 33, 636-647.

Friston, K., Frith, C., Frackowiak, R., \& Turner, R. (1995). Characterizing dynamic brain responses with fMRI: A multivariate approach. NeuroImage, 2, 166-172.

Friston, K., Penny, W., \& Glaser, D. (2005). Conjunction revisited. NeuroImage, 25, 661-667.

Friston, K., Williams, S., Howard, R., Frackowiak, R. S., \& TURNER, R. (1996). Movement-related effects in fMRI time-series. Magnetic Resonance in Medicine, 35, 346-355.

GILBERT, A. M., \& Fiez, J. A. (2004). Integrating rewards and cognition in the frontal cortex. Cognitive, Affective, \& Behavioral Neuroscience, $4,540-552$.

Gray, J. R., \& Braver, T. S. (2002). Personality predicts workingmemory-related activation in the caudal anterior cingulate cortex. Cognitive, Affective, \& Behavioral Neuroscience, 2, 64-75.

Gray, J. R., Burgess, G. C., Schaefer, A., Yarkoni, T., Larsen, R. J., \& Braver, T. S. (2005). Affective personality differences in neural processing efficiency confirmed using fMRI. Cognitive, Affective, \& Behavioral Neuroscience, 5, 182-190.

IкEMOto, S., \& PANKSEPP, J. (1999). The role of nucleus accumbens dopamine in motivated behavior: A unifying interpretation with special reference to reward-seeking. Brain Research Reviews, 31, 6-41.

IVRY, R. (1997). Cerebellar timing systems. International Review of Neurobiology, 41, 555-573.

IVRY, R., \& SPENCER, R. M. (2004). The neural representation of time. Current Opinion in Neurobiology, 14, 225-232.

Koechlin, E., Basso, G., Pietrini, P., Panzer, S., \& Grafman, J. (1999). The role of the anterior prefrontal cortex in human cognition. Nature, 399, 148-151.

Konishi, S., Nakajima, K., Uchida, I., Kikyo, H., Kameyama, M., \& Miyashita, Y. (1999). Common inhibitory mechanism in human inferior prefrontal cortex revealed by event-related functional MRI. Brain, 122, 981-991.

LidDle, P., Kiehl, K. A., \& SMith, A. M. (2001). Event-related fMRI study of response inhibition. Human Brain Mapping, 12, 100-109.

McAvoy, M., Ollinger, J., \& Buckner, R. (2001). Cluster size thresholds for assessment of significant activation in fMRI. NeuroImage, 13, S198.
Meng, X., Rosenthal, R., \& Rubin, D. B. (1992). Comparing correlated correlation coefficients. Psychological Bulletin, 111, 172-175.

Miller, E. K., \& CoHEN, J. D. (2001). An integrative theory of prefrontal cortex function. Annual Review of Neuroscience, 24, 167-202.

Mugler, J. P., \& Brookeman, J. R. (1990). Three-dimensional magnetization-prepared rapid gradient-echo imaging (3D MP RAGE). Magnetic Resonance in Medicine, 15, 152-157.

Nichols, T. E., Brett, M., Andersson, J., Wager, T., \& Poline, J. B. (2005). Valid conjunction inference with the minimum statistic. Neurolmage, 25, 653-660.

O'Doherty, J. P. (2004). Reward representations and reward-related learning in the human brain: Insights from neuroimaging. Current Opinion in Neurobiology, 14, 769-776.

Owen, A. M., McMillan, K. M., Laird, A. R., \& Bullmore, E. (2005). N-back working memory paradigm: A meta-analysis of normative functional neuroimaging studies. Human Brain Mapping, 25, 46-59.

Pardo, J. V., Fox, P. T., \& Raichle, M. E. (1991). Localization of a human system for sustained attention by positron emission tomography. Nature, 349, 61-64.

Pochon, J. B., Levy, R., Fossati, P., Lehéricy, S., Poline, J.-B., PILlon, B., ET AL. (2002). The neural system that bridges reward and cognition in humans: An fMRI study. Proceedings of the National Academy of Sciences, 99, 5669-5674.

Posner, M., \& Petersen, S. (1990). The attention system of the human brain. Annual Review of Neuroscience, 13, 25-42.

Rosvold, H. E., Mirsky, A. F., Sarason, I., Bransome, E. D., Jr., \& BECK, L. H. (1956). A continuous performance test of brain damage. Journal of Consulting Psychology, 20, 343-350.

Rubia, K., Smith, A. B., Brammer, M. J., \& TAYlor, E. (2003). Right inferior prefrontal cortex mediates response inhibition while mesial prefrontal cortex is responsible for error detection. NeuroImage, 20, 351-358.

Small, D., Gitelman, D., Simmons, K., Bloise, S., Parrish, T., \& Mesulam, M. (2005). Monetary incentives enhance processing in brain regions mediating top-down control of attention. Cerebral Cortex, 15, 1855-1865.

SNYDER, A. Z. (1996). Difference image versus ratio image error function forms in PET-PET realignment. In D. Bailer \& T. Jones (Eds.), Quantification of brain function using PET (pp. 131-137). San Diego: Academic Press.

TAlairach, J., \& Tournoux, P. (1988). A co-planar stereotaxic atlas of the human brain. Stuttgart: Thieme.

TaYlor, S. F., Welsh, R. C., Wager, T. D., Phan, K. L., Fitzgerald, K. D., \& Gehring, W. J. (2004). A functional neuroimaging study of motivation and executive function. NeuroImage, 21, 1045-1054.

WAGER, T. D., \& SMITH, E. E. (2003). Neuroimaging studies of working memory: A meta-analysis. Cognitive, Affective, \& Behavioral Neuroscience, 3, 255-274.

Watanabe, M., Hikosaka, K., Sakagami, M., \& Shirakawa, S. (2002). Coding andmonitoring of motivational context in the primate prefrontal cortex. Journal of Neuroscience, 22, 2391-2400.

Woods, R. P., Cherry, S. R., \& Mazziotta, J. C. (1992). Rapid automated algorithm for aligning and reslicing PET images. Journal of Computer Assisted Tomography, 16, 620-633.

Woods, R. P., Grafton, S. T., Holmes, C. J., Cherry, S. R., \& MazziOTTA, J. C. (1998). Automated image registration: I. General methods and intrasubject, intramodality validation. Journal of Computer Assisted Tomography, 22, 139-152.

(Manuscript received April 7, 2006; revision accepted for publication May 10,2007.) 